\title{
SEARCH FOR GIANT CELLS IN THE SOLAR CONVECTION ZONE
}

\author{
B. J. LaBonte and R. Howard \\ Hale Observatories*, Carnegie Institution of Washington
}

The Mount Wilson Observatory has obtained daily full disk digital magnetograms of the Sun since 1966, with 12 to 17 arcsecond resolution. As each magnetogram is taken, the position of the Doppler line shift compensator is also recorded, thus giving a full disk map of the longitudinal velocity. This entire dataset is currently being rereduced on a uniform basis (Howard et al., 1980), and daily arrays of residual velocities are being formed by removing large scale patterns, e.g., Earth's motions, solar rotation, limbshift. Data from the years 1972 through 1978 are used here.

For this study, we have searched for large scale persistent east-west horizontal flows. The procedure is to construct synoptic velocity maps, with the amplitude at each point given by the average of the daily velocities at that point, weighted by the sines of their central meridian distances. This reinforces longitudinal flows and cancels vertical and meridional flows. Since a synoptic (e.g. , Carrington) longitude is visible for many days, random solar velocity noise (supergranules, 5 minute oscillations) is also reduced.

Figure 1 shows two such synoptic maps, for Carrington rotations 1603 and 1606. The velocity pattern during rotation 1603 is random, but during rotation 1606 stripes of alternating velocity sign are seen. A stripe of one sign extends from about $60^{\circ}$ north to $60^{\circ}$ south latitude, with a constant width of about $30^{\circ}$ in longitude. The latitude-averaged velocity amplitude is about $10 \mathrm{~m} \mathrm{~s}^{-1}$. A pair of stripes, east-flowing on the east and west-flowing on the west, appears to be a single outflowing velocity cell. This pattern is similar to that deduced from theory for the largest scale solar convection cells (e.g. , Gilman, 1979). Velocity stripes of this type are seen about $\frac{1}{2}$ the time from 1972 to mid-1974, but only occasionally after that.

Unfortunately these stripes appear to be an instrumental artifact, rather than a solar phenomenon. Stripes of larger amplitude, about 30 to $60 \mathrm{~m} \mathrm{~s}^{-1}$, can occur on a single observation, and leak into the synoptic maps due to

* The Hale Observatories are operated jointly by the Carnegie Institution of Washington and the California Institute of Technology. 

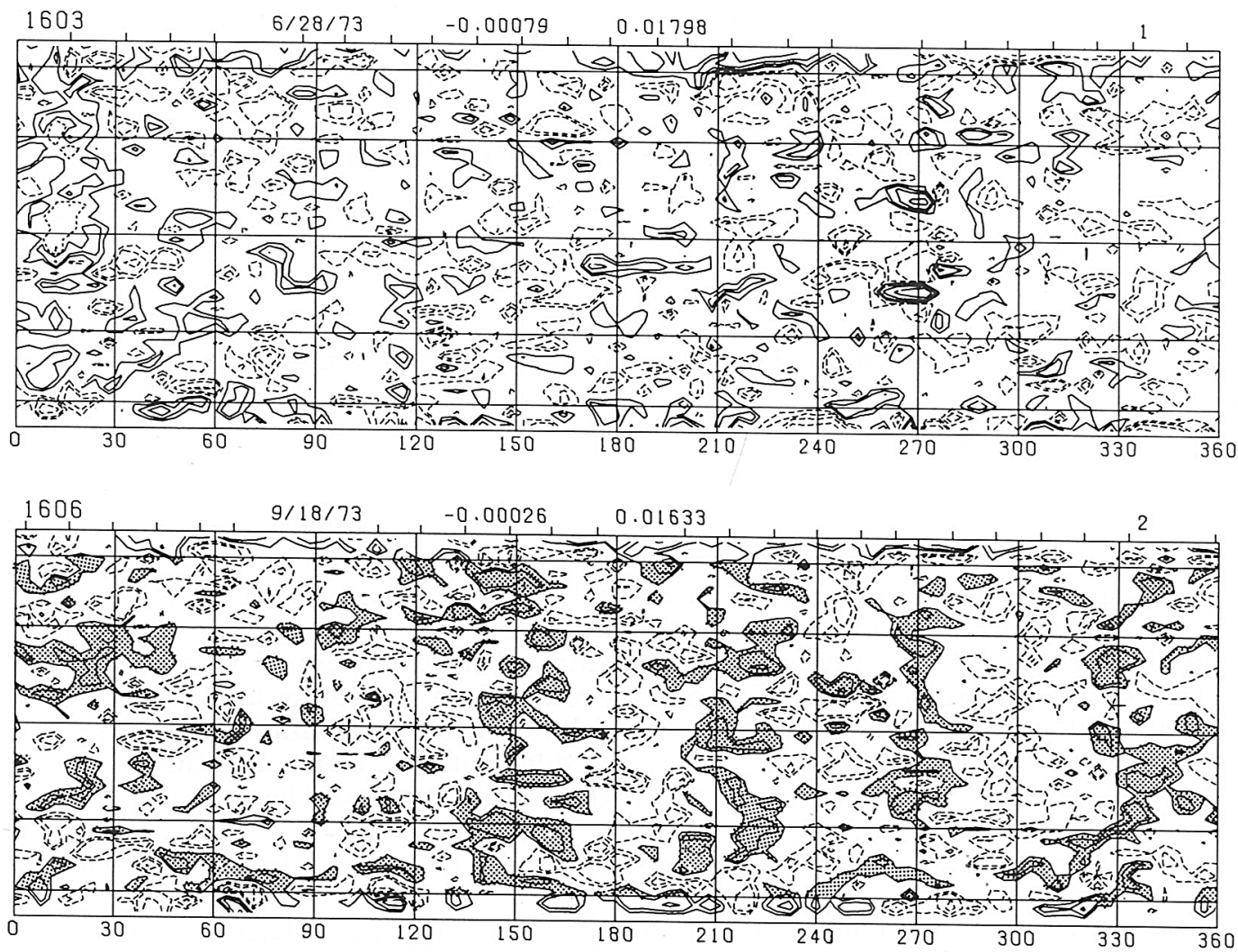

Fig. 1. Synoptic maps of the observed longitudinal velocities on the solar surface, for Carrington rotations 1603 and 1606. Solid contours display eastward flow, dashed contours westward flow. Contour levels are 10,20, 40 and $80 \mathrm{~m} \mathrm{~s}^{-1}$. Eastward velocities have been shaded on the lower map to show the large-scale organization of velocities into stripes.

incomplete sampling or shifts in the daily stripe locations on the solar disk. The cause of the velocity stripes in the daily observations is not yet certain, but is either optical fringes in the spectrum or mechanical problems in the exit slits of the magnetograph.

Because these instrumental stripes are not always present, we can still search for persistent solar motions of this type. Power spectra and autocorrelation functions of the latitude averaged longitudinal velocity have been computed for intervals from 3 months to $3 \frac{1}{2}$ years. Synoptic rotation rates from 0.8 to 1.1 times the Carrington rate have been searched. Figure 2 shows one example of the results, power spectra of the velocity for the two halves of the dataset. The only significant features are the broad peaks in the spectra near 3,6, and 9 cycles (cells) per rotation, which are caused by the instrumental stripes. This result is true of all the analyses thus far. We can set upper limits on the amplitude of a true solar cell-like velocity pattern which are quite small. A broad spectral feature ( $>1$ cycle per 

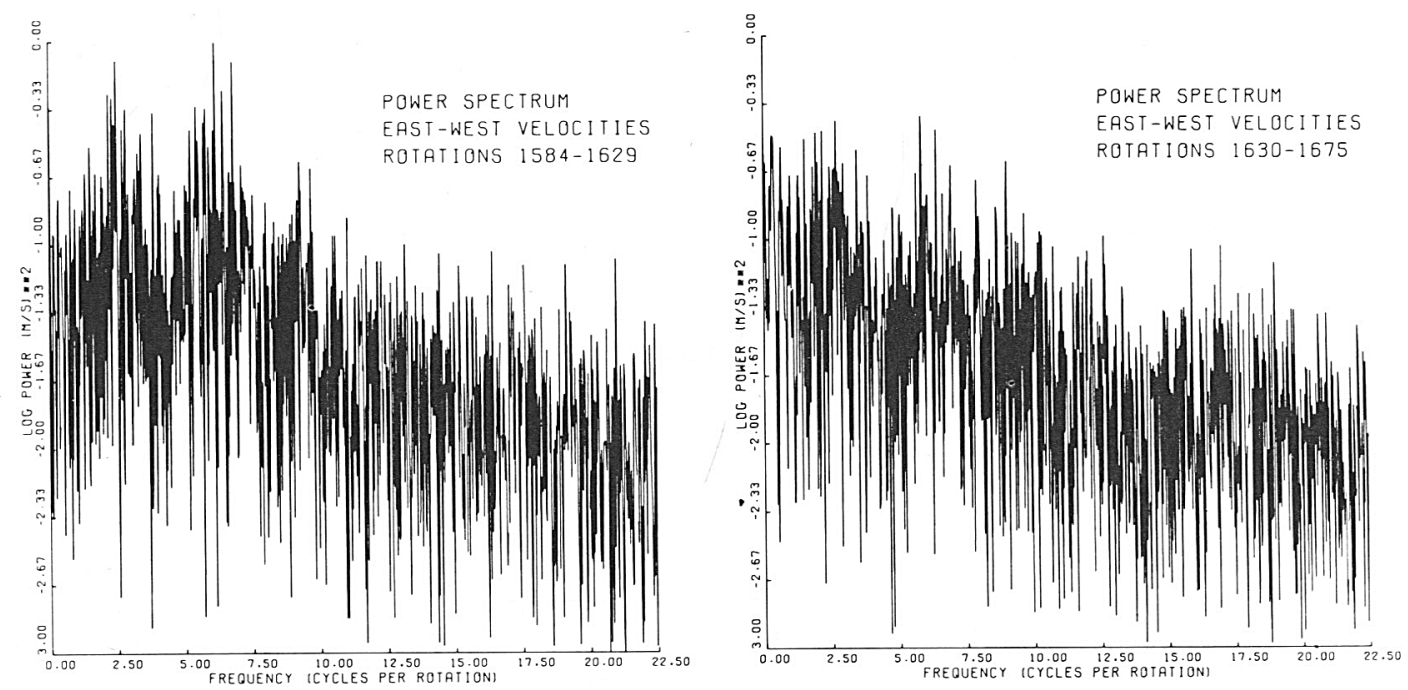

Fig. 2. Power spectra of the latitude-averaged longitudinal velocity, for Carrington rotations 1584 to 1629 , and 1630 to 1675 . Broad peaks at 3,6 , and 9 cycles per rotation in the first spectrum are caused by the instrumental velocity stripes. Their amplitude is much reduced in the second spectrum, for a time interval during which stripes were rare. The integrated amplitude (RMS) of the instrumental stripes is only $\approx 5 \mathrm{~m} \mathrm{~s}^{-i}$, and any solar features must be weaker.

rotation) must have an amplitude $\leqslant 5 \mathrm{~m} \mathrm{~s}^{-1}$ for lifetimes 1 year, and $\leqslant 10 \mathrm{~m} \mathrm{~s}^{-1}$ for lifetimes of a few months. A narrow spectral feature $(<1$ cycle per rotation) must be $\leqslant 2 \mathrm{~m} \mathrm{~s}^{-1}$ for lifetimes 1 year, and $\leqslant 5 \mathrm{~m} \mathrm{~s}^{-1}$ for lifetimes of a few months. These limits apply at all frequencies between 2 and 20 cycles per rotation.

\section{RE FERENCES}

Gilman, P. A.: 1979, Astrophys. J., 231, pp. 284-292. Howard, R., Boyden, J. E., and LaBonte, B. J.: 1980,66 , pp. 167 . Solar Phys.

\section{DISCUSSION}

Bratenahl: Yoshimura's global convection cells, I believe, have a retrograde motion. Did you research for that possibility?

LaBonte: Yes. By analyzing the data while using a variety of rotation rates, we have allowed for the possibility that the convective cells slip with respect to the surface or the core. 